\title{
Integration not a solution for frequent ED use
}

\author{
Cite as: CMAJ 2017 January 16;189:E92-3. doi: 10.1503/cmaj.109-5353
}

$\mathrm{E}$ fforts to reduce emergency department (ED) visits among frequent users may be misguided, particularly when it comes to patients with mental illnesses and addictions, say researchers.

Increasing costs and overcrowding have propelled projects to encourage these frequent flyers to land elsewhere in the health system, usually by referring them to case managers who act as bridges to services outside the hospital. This approach initially appeared to reduce ED visits in observational studies.

But now more rigorous trials are beginning to tell a different story, says Dr. Vicky Stergiopoulos, a clinician scientist at the Centre for Research on Inner City Health in Toronto. "It's a myth that integration is the solution to it all," she argued at the recent Canadian Psychiatric Association conference.

In her study of 166 frequent ED users at six Toronto hospitals, Stergiopoulos found that case management didn't affect ED use compared to a control group. Participants had visited the ED five or more times in the past year, and had at least one visit related to mental illness or substance use.

"We saw reductions over time in ED use, but the reductions in the intervention and control group were comparable," Stergiopoulos said. If the control group wasn't factored in, "we would have looked phenomenally successful."

In theory, patients are doing themselves a disservice by seeking piecemeal care in EDs when primary care and community services are better equipped to manage chronic conditions. "We had hypothesized that this population was not connected to services in the community, and if we offered them case management it would decrease their use of ED," Stergiopoulos said.

But that hypothesis proved wrong. Many study participants were over-connected, with links to multiple community services and even case managers. "Getting all these

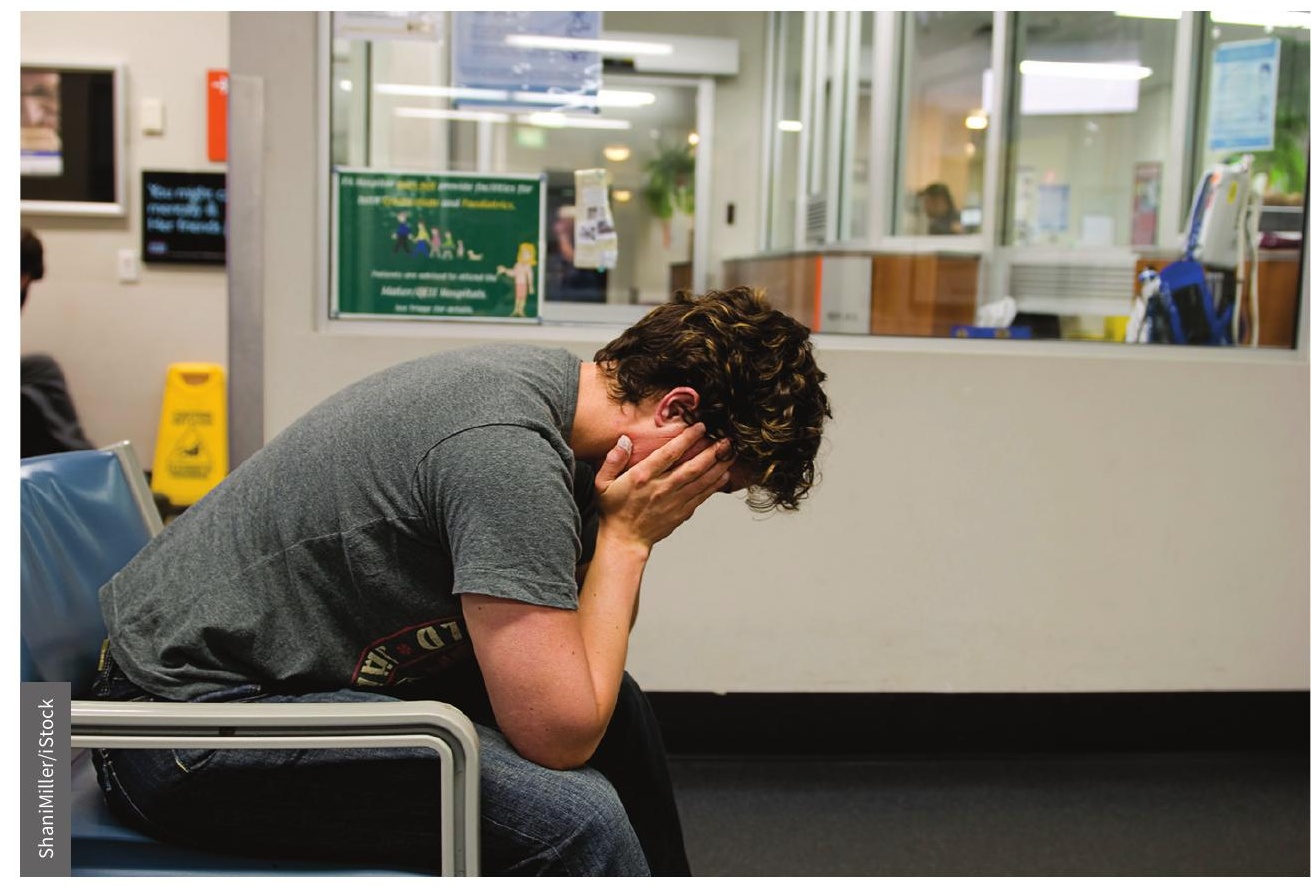

Frequent users of emergency departments have complex needs that aren't being met in the community.

supports to speak to each other and coordinate care was very challenging," Stergiopoulos said. It also raised a new question: If patients had access to more appropriate services, why were they going to the ED?

The study showed several good reasons. "This population is actually quite sick," Stergiopoulos explained. "The vast majority had three or more chronic physical conditions in additional to their mental health conditions." She was surprised by the high number of deaths among participants, whose average age was in the mid40s. "Nine deaths among 166 young people gives you the severity and complexity of the needs."

In addition, participants told researchers they had nowhere else to go. They faced long waits for primary care and community services, and busy signals when they called crisis hotlines. Even when they did access care, they were often sent back to the ED.

"We heard loud and clear that existing services would not meet their needs," Stergiopoulos said.

This included "huge quality problems" in the case management provided by community mental health agencies, which in some cases "was so poor it was a safety risk," she said. "Case managers, who maybe had two years of training after high school, are thrown out with no additional training or supervision to manage some of the most complex needs in our city."

It's not clear that intervention is even necessary, Stergiopoulos added. Looking at patterns of ED use across Ontario, researchers discovered that only one in four patients remained heavy users from one year to the next.

Dr. Roger Bland, professor emeritus in the department of psychiatry at the University of Alberta, said a project in his province to reduce ED visits by frequent users was ultimately dropped "because they weren't a constant population.” 
"The really heavy users tended to be people with major medical problems and they didn't stay heavy users; they either died or things improved," he explained.

According to Stergiopoulos, these and other failed projects have started a pendulum swing away from "stop-gap interventions that connect ED with the community." She was involved in evaluations of nine integration projects funded by the Ontario government and nearly 40 by the Ontario Medical Association. "None of them had positive outcomes."

According to Deb Wise Harris, the research coordinator for the Toronto case- management study, hospitals should refocus on improving ED care for repeat patients. Study participants were often met with impatience and "surface-level or hurried care" from hospital staff, Wise Harris said. In some cases, they were dismissed or kicked out. "There is a perception among health care staff that higher patient satisfaction could encourage ED users to keep coming back, but in fact some research suggests the opposite."

She cited a 1995 randomized trial in The Lancet that found homeless patients were more satisfied with ED visits and less likely to return if they received compassionate care from volunteers. This included simple gestures like asking if a patient needed something to eat or drink. "Patients tend to return frequently until they are satisfied with their treatment," the study concluded.

Wise Harris argued that interventions to reduce ED staff burnout and compassion fatigue could be a win-win for hospitals and patients. "Increasing ED staff sensitivity towards frequent users with mental health and addictions concerns and improving referrals to timely aftercare may improve patient satisfaction and reduce recidivism."

Lauren Vogel, CMAJ 